\title{
ECE20]6 Antithyroid arthritis syndrome
}

Anne-Cécile Paepegaey, Maria Mavromati, Philippe Chanson, Sylvie Salenave.

Assistance Publique Hôpitaux de Paris, Hôpital de Bicêtre, Service d'Endocrinologie et des maladies de la Reproduction, Le Kremlin-Bicêtre, France

\section{Background:}

The most frequent minor adverse antithyroid drug reactions are skin reactions $(4$ to $6 \%$ ), gastrointestinal effects ( 1 to $5 \%$ ) and arthralgias (1 to $5 \%$ ) and the most frequent major adverse antithyroid drug reactions are antithyroid arthritis syndrome (1 to $2 \%)$, agranulocytosis $(0.1$ to $0.5 \%)$ and hepatitis $(0.1$ to $0.2 \%)$. Major adverse drug reactions occur rarely but cause the stop of all antithyroid drugs.

\section{Clinical case:}

We describe the case of a 20 -year-old woman with Graves' disease.

Two weeks after the beginning of carbimazole therapy ( $60 \mathrm{mg} /$ day), she developed an urticarial reaction that led to discontinuation of treatment.

Nevertheless, diffuse aching limbs appeared abruptly twenty four hours after stopping the treatment

Due to the lack of clinical improvement, our patient was admitted to the hospital. She had skin reaction with itching, skin rash (figure 1), dermatographic urticaria (figure 2) and arthralgia.

Treatment with antihistamin drugs and analgesics has been started but arthralgia progressed to migratory oligoarthritis and body temperature increased to $38^{\circ} \mathrm{C}$.

Blood tests only showed inflammatory syndrome. Creatine kinase level, joint $X$ rays, left knee joint viral serologies, ANCA antibodies, rheumatoid factor, anti-nuclear antibodies, anti CCP antibodies and anti SSA/RO antibodies were normal.

The patient was treated with anti-histamin and non-steroidal anti-inflammatory drugs as well as corticosteroids and clinical improvement occurred a few weeks later with no recurrence of skin rash or arthralgia.

Hyperthyroidism was treated with 131 iodine.

\section{Discussion:}

Polyarthritis is a rare major side effect of antithyroid drugs. It is important to distinguish anti thyroid arthritis syndrome from minor arthralgia. Contrary to minor arthralgia, anti thyroid arthritis syndrome requires treatment discontinuation, due to cross reactions between the different medications. The majority of polyarthritis cases due to antithyroid treatment described in the literature appear in an autoimmune context [1].

Here we described a rare case of antithyroid arthritis syndrome without evidence of autoimmunity.

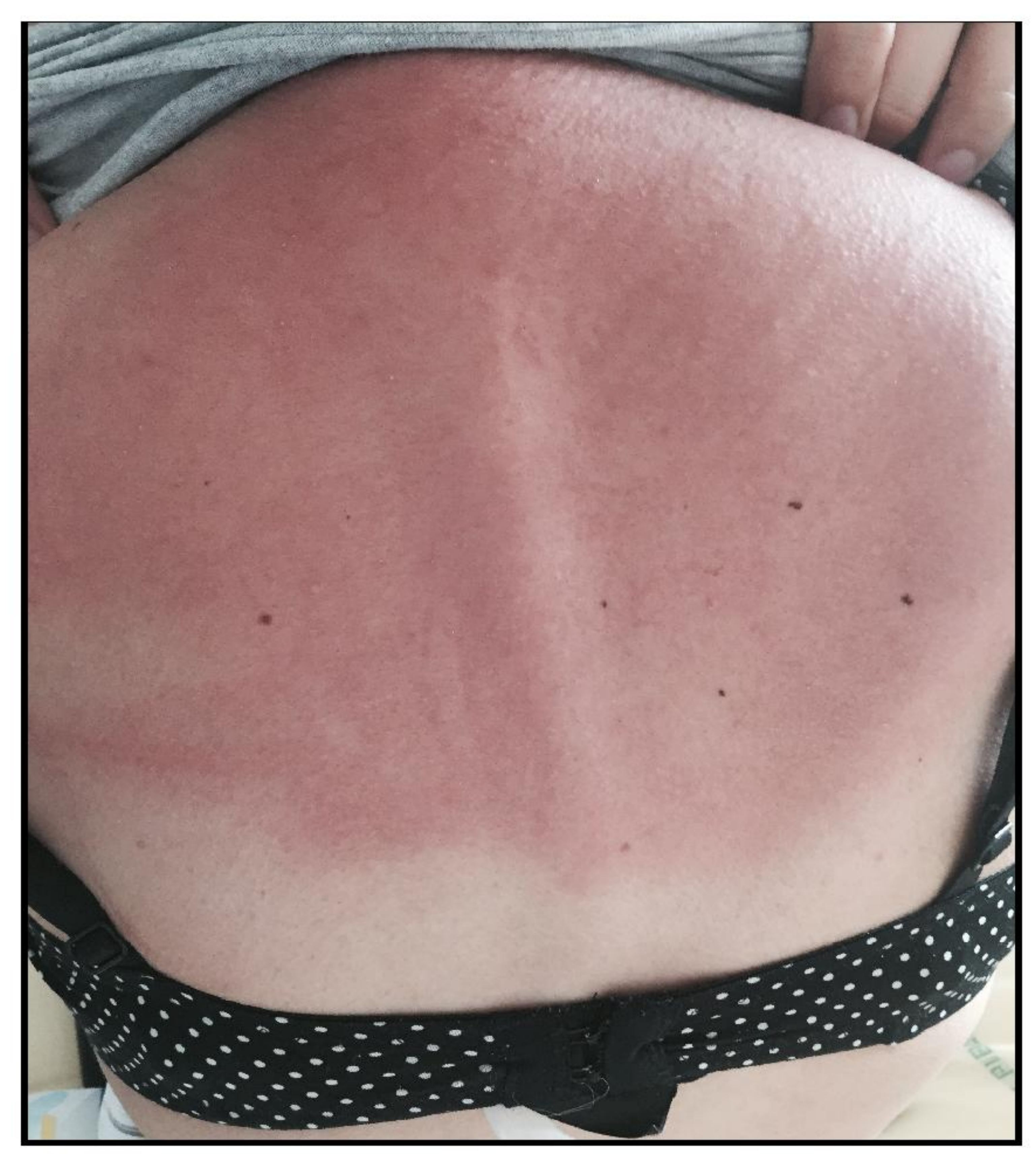

Figure 1. Skin rash

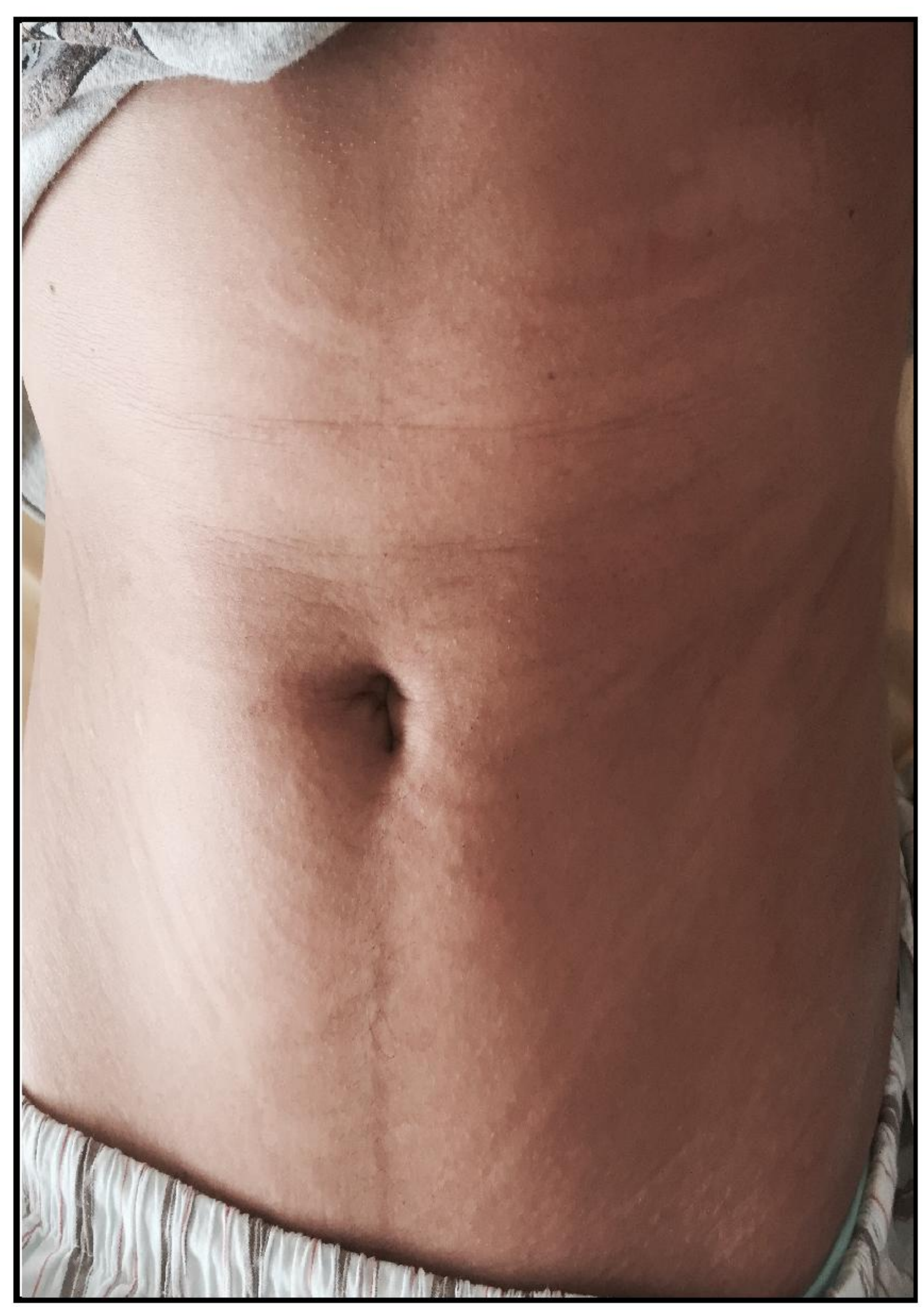

Figure 2. Dermatographic urticaria

Conclusion: In conclusion, it seems important to well-known this side effect of antithyroid drug, in order to stop antithyroid drug treatment, to use a radical treatment for hyperthyroidism and to use steroidal anti-inflammatory or corticosteroids to treat polyarthritis. 